\title{
Identification of As, Ge and Se Photoluminescence in GaN Using Radioactive Isotopes
}

\author{
A. Stötzler ${ }^{1}$, R. Weissenborn ${ }^{1}$, M. Deicher ${ }^{1}$, and ISOLDE Collaboration ${ }^{2}$ \\ ${ }^{1}$ Fakultät für Physik, Universität Konstanz, D-78457 Konstanz, Germany \\ ${ }^{2}$ CERN / PPE, CH-1211 Geneva 23, Switzerland
}

\begin{abstract}
We report on experiments which unequivocal identify the chemical nature of optical transitions related to As $(2.58 \mathrm{eV})$, Ge $(3.398 \mathrm{eV})$ and $\mathrm{Se}(1.49 \mathrm{eV})$ found in the photoluminescence (PL) spectra of GaN. For this purpose epitaxial GaN layers were doped by ion implantation $\left(60 \mathrm{keV}, 3 \times 10^{12} \mathrm{~cm}^{-2}\right)$ with the radioactive isotopes ${ }^{71} \mathrm{As}$ and ${ }^{72} \mathrm{Se}$. The isotope ${ }^{71} \mathrm{As}$ (half-life $64.28 \mathrm{~h}$ ) decays first into ${ }^{71} \mathrm{Ge}(11.43 \mathrm{~d}$ ), which finally transmutes into stable ${ }^{71} \mathrm{Ga}$. The isotope ${ }^{72} \mathrm{Se}$ decays via ${ }^{72} \mathrm{As}(26 \mathrm{~h})$ into stable ${ }^{72} \mathrm{Ge}$. These chemical transmutations were monitored with photoluminescence spectroscopy (PL). The half-lives resulting from exponential fits on our PL data are in excellent agreement with the half-lives of the isotopes. Our experiments clearly show that in each case the luminescence center involves exactly one As, Ge or Se atom. In addition to this, the results imply that no optically active $\mathrm{Ga}_{\mathrm{N}}$ antisite exists.
\end{abstract}

\section{INTRODUCTION}

$\mathrm{GaN}$ is a promising material for the construction of blue laser diodes, ultrahigh power switches or UV photo detectors and much information about the growing of $\mathrm{GaN}$ and device processing was gathered during the last years and is summarized in recent published review articles [1,2]. Ge and Se are promising candidates to act as donors in $\mathrm{GaN}$, and calculations predict a much higher doping efficiency for $\mathrm{Ge}$ than $\mathrm{Si}$, the commonly used donor in $\mathrm{GaN}$ [3,4]. Arsenic is suspected to compensate charge carriers after ion implantation [5], in spite of being isoelectronic to N. The energy levels of these impurities have been first optically determined by Pankove et al. [6]. As was found to produce a broad PL band centered at $2.58 \mathrm{eV}$, in agreement with results from Metcalfe et al. [5] and Li et al. [7]. A deep PL band at $1.59 \mathrm{eV}$ was assigned to Se, but recent results only report on enhanced DAP recombination at $3.278 \mathrm{eV}$ [8] or an increasing yellow luminescence [9] after doping GaN with Se. An increase of the UV emission (3.2 eV) and the yellow luminescence $(2.2 \mathrm{eV})$, but no additional transitions, was observed in Ge doped or implanted samples $[6,10]$. But one has to take into account that PL spectroscopy is not able to determine the chemical nature of a defect. Hence, a chemical identification of a defect is difficult and the assignments are sometimes controversial. One way out of this dilemma is to use element specific properties, like the half-life of a radioactive isotope undergoing a chemical transmutation. If an optical transition is due to a defect in which the parent or daughter isotope is involved, the concentration of that defect will change with the half-life of the radioactive decay. Finally, the change in the defect concentration then shows up in the PL intensity of the corresponding transition [11,12].

Another important class of intrinsic defects in III-V semiconductors is antisites. Theoretical investigations of Jenkins et al. calculate the $\mathrm{Ga}_{\mathrm{N}}$ antisite level at $0.7 \mathrm{eV}$ above the valence band [13], in agreement with the predictions of Tansley et al. [14]. In contrast 
to these results, Boguslawski et al. [15] calculate the $\mathrm{Ga}_{\mathrm{N}}$ antisite level at around $\mathrm{E}_{\mathrm{V}}+1.4$ $\mathrm{eV}$, and Neugebauer et al. state that antisites in $\mathrm{Ga}_{\mathrm{N}}$ are energetically less favorable [16] than in other III-V semiconductors. As shown by Magerle et al. [17], another advantage of radioactive isotopes in PL experiments is the chance to create antisites intentionally with suitable isotopes. We report on experiments with the isotope ${ }^{71} \mathrm{As}$, which decays via ${ }^{71} \mathrm{Ge}$ into stable ${ }^{71} \mathrm{Ga}$. Provided that after implantation and annealing all ${ }^{71} \mathrm{As}$ occupies a $\mathrm{N}$ site and no site changes are taking place during the decay, then all ${ }^{71} \mathrm{Ga}$ atoms form $\mathrm{Ga}_{\mathrm{N}}$ antisite defects. The aim of this report is the unequivocal chemical assignment of optical transitions found in $\mathrm{As}, \mathrm{Ge}$, and Se doped $\mathrm{GaN}$, and to investigate the existence of optically active $\mathrm{Ga}_{\mathrm{N}}$ levels.

\section{EXPERIMENTAL}

Nominally undoped $\mathrm{GaN}$ layers $\left(\mathrm{n} \approx 5 \times 10^{16} \mathrm{~cm}^{-3}\right)$ grown by metal organic vapor phase epitaxy (MOVPE) on AlN/c-sapphire substrate by Cree Research were implanted either with radioactive ${ }^{72} \mathrm{Se}$ or ${ }^{71} \mathrm{As}$. The implantations were carried out at the on-line mass separator ISOLDE at CERN with an energy of $60 \mathrm{keV}$ and a dose of $3 \times 10^{12}$ ions $/ \mathrm{cm}^{2}$. The ions end up within a Gaussian shaped profile centered at $21 \mathrm{~nm}$ depth with a width of $10 \mathrm{~nm}$ and a peak concentration of about $2 \times 10^{18} \mathrm{~cm}^{-3}$. To serve as reference, a small part of each sample was not implanted. The implantation induced damage was reduced by annealing the samples at $1270 \mathrm{~K}$ for $10 \mathrm{~min}$ in sealed quartz ampoules filled with nitrogen gas at a pressure of 1 bar at room temperature. The isotope ${ }^{72} \mathrm{Se}$ transmutes via the decay chain ${ }^{72} \mathrm{Se}(8.4 \mathrm{~d}) \rightarrow{ }^{72} \mathrm{As} \rightarrow(26 \mathrm{~h})$ into stable ${ }^{72} \mathrm{Ge}$ while the isotope ${ }^{71} \mathrm{As}$ $(64.28 \mathrm{~h})$ first decays into ${ }^{71} \mathrm{Ge}(11.43 \mathrm{~d})$, which finally transmutes into stable ${ }^{71} \mathrm{Ga}$. The half-life of each decay is given in parentheses. These chemical transmutations were monitored by PL spectroscopy at $4 \mathrm{~K}$ using a He flow cryostat. The $325 \mathrm{~nm}$ line of a $\mathrm{HeCd}$-laser with an excitation density of $160 \mathrm{Wcm}^{-2}$ was used to excite the samples. The luminescence was dispersed with a $0.75 \mathrm{~m}$ monochromator and detected with a cooled GaAs-photomultiplier.

\section{RESULTS AND DISCUSSION}

Figure 1a shows a selection of the 22 recorded PL spectra of ${ }^{71}$ As-doped GaN successively taken within 56 days after ion implantation and annealing. The spectra are not corrected for the spectral response of the measurement system. The common features of all spectra are the transition DX at $3.471 \mathrm{eV}$ resulting from a donor-bound exciton [18] and the broad band centered at $2.2 \mathrm{eV}$ labeled with YL, known as the "yellow luminescence" in GaN [9]. The spectrum recorded 12 hours after implantation shows clearly a new broad and intense PL band centered at $2.58 \mathrm{eV}$, in contrast to the unimplanted part of the sample (not shown). In addition, a new transition labeled with $\mathrm{Ge}$ at $3.398 \mathrm{eV}$ and its LO phonon replica Ge-LO at $3.306 \mathrm{eV}$ can be seen. The intensity of the PL band at 2.58 $\mathrm{eV}$ decreases continuously during the whole measuring period, in contrast to the intensities of the PL transitions at $3.398 \mathrm{eV}$ and $3.306 \mathrm{eV}$. Within the first eight days after implantation the intensity of these transitions increases before this trend changes into a decrease of the PL intensity of these lines after nine days. Finally, after 56 days no luminescence at $2.58 \mathrm{eV}$ and only weak luminescence at $3.398 \mathrm{eV}$ can be detected. A comparison with the radioactive decay chain of the isotope ${ }^{71}$ As clearly shows that the decreasing PL band at $2.58 \mathrm{eV}$ has to be correlated with As, in agreement with earlier assignments [5-7]. 
The transition at $3.398 \mathrm{eV}$ and its phonon replica can only be explained by a recombination center involving Ge. A detailed analysis of the time dependence of the PL intensities of the As and Ge transitions delivers information about the involved number of As or Ge atoms, respectively. For such an analysis, it is necessary to eliminate intensity variations due to inhomogeneous samples or experimental limitations, like focusing reproducibly onto the entrance slit of the monochromator, by a suitable normalization. The selection of a normalization point requires that the normalization point itself shows no time dependency and does not overlap with the questioned transitions. For this reason, all spectra were normalized to the same intensity at $1.59 \mathrm{eV}$.
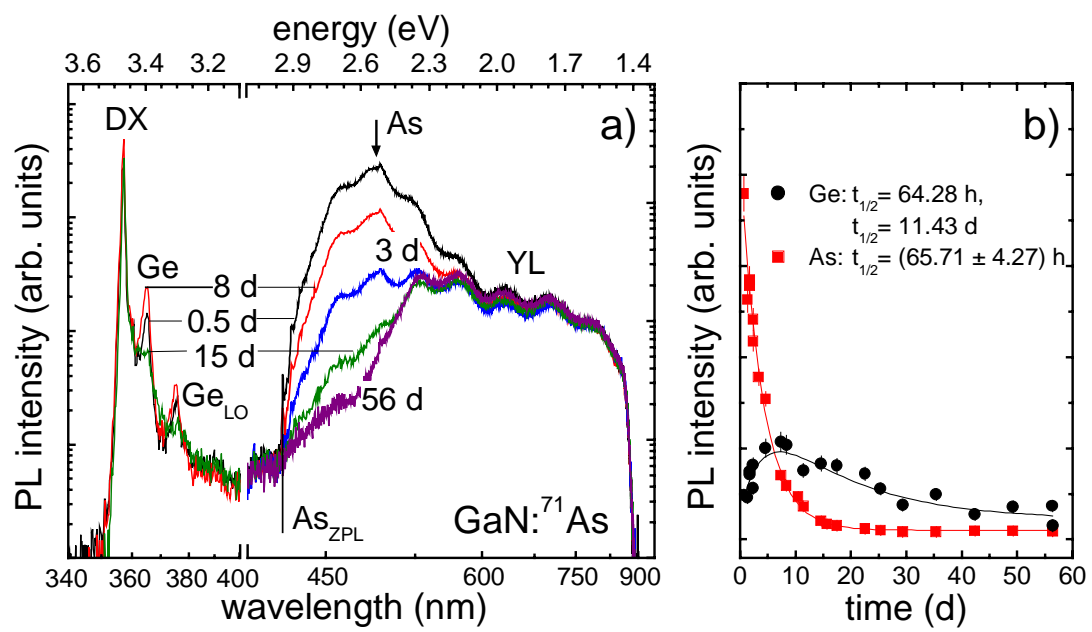

Figure 1. a) PL spectra of ${ }^{71} A$ s doped GaN recorded at $4 \mathrm{~K}$ between $0.5 \mathrm{~d}$ and $56 \mathrm{~d}$ after implantation and annealing. All spectra are normalized to the intensity at $1.59 \mathrm{eV}$. b) Normalized PL intensity of the As (squares) and Ge (circles) related transitions in GaN as a function of time. The solid lines correspond to exponential fits to the data using equations 1 and 2.

In figure $1 b$, the integral PL intensities of the As and Ge related PL transitions are plotted as a function of time. The solid lines correspond to exponential fits to the data using equations 1 and 2 :

$$
\begin{gathered}
I^{A s}(t)=I_{0}^{A s} e^{\left((-\ln 2) t / t_{1 / 2}\right)} \\
I^{G e}(t)=I_{0}^{A s} t_{1 / 2}^{G e}\left(t_{1 / 2}^{A s}-t_{1 / 2}^{G e}\right)^{-1}\left(e^{\left((-\ln 2) t / t_{1 / 2}^{A s}\right)}-e^{\left((-\ln 2) t / t_{1 / 2}^{G e}\right)}\right)+I_{0}^{G e} e^{\left((-\ln 2) t / t_{1 / 2}^{G e}\right)}
\end{gathered}
$$


For the As-band the fit yields a half-life of $t_{1 / 2}^{A s}=(65.71 \pm 4.27) \mathrm{h}$, in very good agreement with the nuclear half-life of ${ }^{71} \mathrm{As}\left(\mathrm{t}_{1 / 2}=64.28 \mathrm{~h}\right)$. The values generated by equation 2 applied to the Ge related transitions are very sensitive to the ratio of the half-lives. Due to the few data points in the maximum region, it was not possible to fit the experimental values in a significant way. For this reason the half-lives were fixed to their theoretical values, and only the constants $I_{0}^{A s}$ and $I_{0}^{G e}$ were fitted to the experimental values in the case of the Ge related transition. As shown in figure $1 \mathrm{~b}$, the behavior of the experimental values is very well described using the known half-lives of As and Ge. This result leads to the conclusion that both the As band centered at $2.58 \mathrm{eV}$ and the Ge transition at $3.398 \mathrm{eV}$ are caused by recombination centers containing only one As or Ge atom, respectively. Additionally, these results call into question some theoretical predictions for the anti-site $\mathrm{Ga}_{\mathrm{N}}$. One can assume that after annealing, all ${ }^{71} \mathrm{As}$ nuclei were placed on $\mathrm{N}$ lattice sites, since As is isolectronic to $\mathrm{N}$ in GaN. If one further assumes that no site changes will take place due to the decay to $\mathrm{Ga}$, then all $\mathrm{Ga}$ atoms end up on a $\mathrm{N}$ site and therefore form antisites. The level of the antisite $\mathrm{Ga}_{\mathrm{N}}$ has been calculated to be approximately $2.8 \mathrm{eV}[13,14]$. The fact that no additional transitions were detected may be evidence for an incorrectly determined energy of the anti-site $\mathrm{Ga}_{\mathrm{N}}$. A few PL spectra were recorded with a $\mathrm{Ge}$ detector, and they also show no additional transitions between $0.7 \mathrm{eV}$ $-1.3 \mathrm{eV}$. But we want to point out that this conclusion is not compelling. If one takes into account the atomic radius of As compared to those of $\mathrm{N}$ or $\mathrm{Ga}$, respectively, it is more probable that As is incorporated on Ga sites. Additionally, the recoil energy transferred to the ${ }^{71} \mathrm{Ge}$ nucleus $(30 \mathrm{eV})$ is large enough to displace a fraction of the atoms from their lattice sites. It is also possible that the $\mathrm{Ga}_{\mathrm{N}}$ antisite level is optically inactive.

An additional experiment was performed to determine if Se creates an optically active recombination center and to check again the previous results. A GaN sample was doped with ${ }^{72} \mathrm{Se}$, which decays via ${ }^{72} \mathrm{As}$ into stable ${ }^{72} \mathrm{Ge}$. Figure 2 a shows four of the 15 PL spectra of the ${ }^{72} \mathrm{Se}$ doped GaN recorded within 50 days after implantation and annealing. As in the previous experiment, all spectra have the DX transition and the yellow luminescence band YL in common. The spectrum recorded one day after implantation shows a new PL band centered at $1.49 \mathrm{eV}$. Also, the same PL transitions are observed as in the previous experiment, namely the As band centered at $2.58 \mathrm{eV}$ and the Ge transition at $3.398 \mathrm{eV}$ and its phonon replica (Ge-LO). All spectra have been normalized to the PL intensity at $1.83 \mathrm{eV}$. Within the 50 days, the PL intensity of the $1.49 \mathrm{eV}$ transition and the intensity of the As band are decreasing while the intensities of the Ge related transitions (Ge and Ge-LO) are increasing continuously. Also these results clearly show the involvement of $\mathrm{As}$ in the $2.58 \mathrm{eV}$ band and $\mathrm{Ge}$ in the $3.398 \mathrm{eV}$ transition. Furthermore, the 1.49 $\mathrm{eV}$ emission has to be caused by a recombination center involving Se. Since As is the daughter isotope of Se, one expects that the intensity of the As band increases first, and decreases after the maximum As concentration is reached. Here, the As band starts to decrease immediately without a preceding increase. This result is not surprising, since during the ${ }^{72} \mathrm{Se}$ implantation a fraction of ${ }^{72} \mathrm{As}$ atoms was co-implanted due to the decay of ${ }^{72} \mathrm{Se}$ in the implantation ion source itself. Hence, ${ }^{72} \mathrm{As}$ and even ${ }^{72} \mathrm{Ge}$ are still present in the ion beam and cannot be separated by the separation magnet. One also has to keep in mind that the half-life of As is much smaller than the half-life of Se. After two days, the initial implanted As concentration can be neglected, leading to the same time constant $\left(t_{1 / 2}^{S e}=8.4 \mathrm{~d}\right)$ for the As concentration resulting from Se decays and Se itself. 
Figure $2 b$ shows the normalized PL intensities of the Se, As, Ge and Ge-LO transitions as a function of time after implantation. The solid lines represent exponential fits to the data using fitting functions similar to equation 1 . The fits yield half-lives of $t_{1 / 2}^{S e}=$

$(8.55 \pm 0.62) \mathrm{d}, t_{1 / 2}^{A s}=(8.48 \pm 0.25) \mathrm{d}, t_{1 / 2}^{G e}=(8.26 \pm 0.95) \mathrm{d}$, and $t_{1 / 2}^{G e-L O}=(8.39 \pm 0.95) \mathrm{d}$ which perfectly agree with the expected ones. As in the previous experiment, the recombination centers responsible for the Ge and As transitions contain exactly one Ge or As atom, respectively, and the same conclusion applies to Se, too. In both experiments, identical PL transitions for As were observed, therefore, in both cases the As atom is placed on the same lattice site. Se is known to replace $\mathrm{N}$ atoms in $\mathrm{GaN}$ [9], and the recoil energy during the decay of ${ }^{72} \mathrm{Se}$ to ${ }^{72} \mathrm{As}(0.83 \mathrm{eV})$ is not sufficient for a site change. Therefore, As is also incorporated on a $\mathrm{N}$ site. This supports the recombination mechanism proposed by Li et al. [7], who claim that the As band is due to excitons bound to isoelectronic impurities.
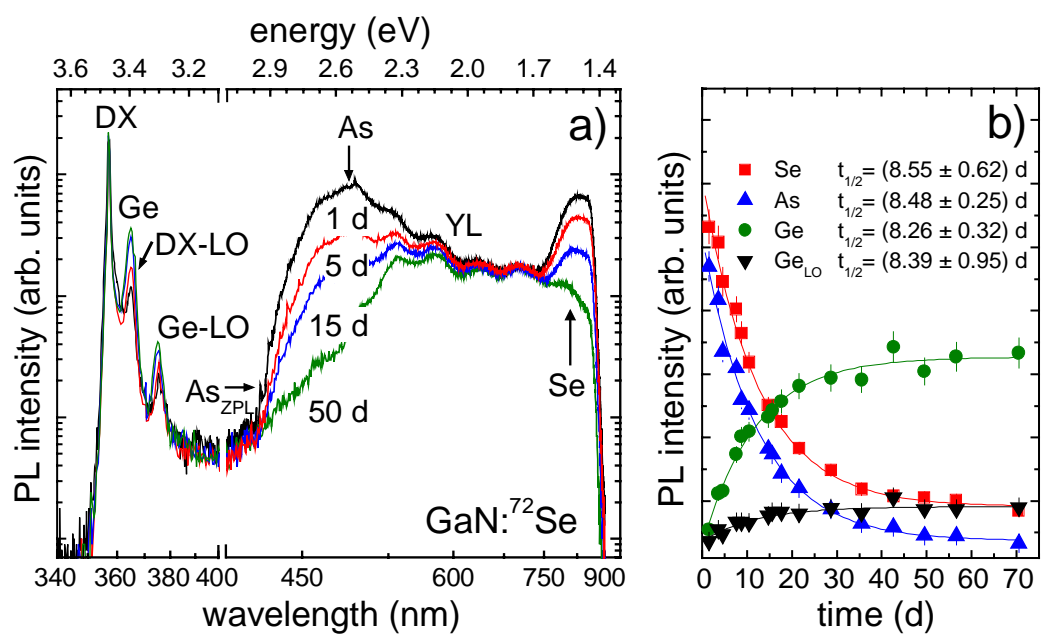

Figure 2. a) PL spectra of ${ }^{72}$ Se doped GaN recorded at $4 \mathrm{~K}$ between $1 \mathrm{~d}$ and $50 \mathrm{~d}$ after implantation and annealing. All spectra are normalized to the intensity at $1.83 \mathrm{eV}$. b) Normalized photoluminescence intensity of the Se (squares), As (up triangles), Ge (circles), and Ge-LO (down triangles) transitions in GaN as a function of time. The solid lines correspond to exponential fits using equation 1.

In figures 1a and 2a, a small peak denoted with $\mathrm{As}_{\mathrm{ZPL}}$ at $2.945 \mathrm{eV}$ can be detected, followed by a series of less well resolved peaks, each separated by the energy of a LO phonon $(92 \mathrm{meV})$ on the high energy side of the As band. This substructure is additional evidence for this assignment. Unfortunately, the recoil energy $(140 \mathrm{eV})$ transferred to the ${ }^{72} \mathrm{Ge}$ nucleus is again sufficient for a site change of the ${ }^{72} \mathrm{Ge}$. Furthermore, our results do not agree with the $\mathrm{Ge}_{\mathrm{N}}$ level $\left(\mathrm{E}_{\mathrm{V}}+0.4 \mathrm{eV}\right)$ calculated by Jenkins et al. [13], which is too high, compared with our results. Hence, it is not yet definitely clear if the Ge transition is caused by a $\mathrm{Ge}_{\mathrm{Ga}}$ or $\mathrm{Ge}_{\mathrm{N}}$ defect. 


\section{CONCLUSION}

In conclusion, using radioactive isotopes we have proven that the PL emission centered at $2.58 \mathrm{eV}$ is caused by a recombination center involving one As atom located on a $\mathrm{N}$ site. Se is found to produce a luminescence band centered at $1.49 \mathrm{eV}$ and $\mathrm{Ge}$ introduces a PL transition at $3.398 \mathrm{eV}$. In both cases, only one Se or Ge atom, respectively, is involved in the defect center. Furthermore, we do not observe any transition related to the $\mathrm{Ga}_{\mathrm{N}}$ antisite.

\section{ACKNOWLEDGEMENT}

This work has been supported by the Bundesminister für Bildung, Wissenschaft Forschung und Technologie under Grant No. 03-DE5KO1-6.

\section{REFERENCES}

[1] S.J. Pearton, J.C. Zolper, R.J. Shul, and F. Ren, J. Appl. Phys. 86, 1 (1999).

[2] O. Ambacher, J. Phys. D: Appl. Phys. 31, 2653 (1998).

[3] C.H. Park and D.J. Chadi, Phys. Rev. B 55, 12995 (1997).

[4] P. Boguslawski and J. Bernholc, Phys. Rev. B 56, 9496 (1997).

[5] R.D. Metcalfe, D. Wickenden, and W.C. Clark, J. Lumin. 16, 405 (1978).

[6] J.I. Pankove and J.A. Hutchby, J. Appl. Phys. 47, 5387 (1976).

[7] X. Li, S. Kim, E.E. Reuter, S.G. Bishop, and J.J. Coleman, Appl. Phys. Lett. 72, 1990 (1998).

[8] G. Yi and B.W. Wessels, Appl. Phys. Lett. 69, 3028 (1996).

[9] H.M. Chen, Y.F. Chen, M.C. Lee, and M.S. Feng, Phys. Rev. B 56, 6942 (1997)

[10] S. Nakamura, T. Mukai, and M. Senoh, Jpn. J. Appl. Phys. 31, 2883 (1992).

[11] R. Magerle, A. Burchard, M. Deicher, T. Kerle, W. Pfeiffer, and E. Recknagel, Phys. Rev. Lett. 75, 1594 (1995).

[12] A. Stötzler, R. Weissenborn, M. Deicher, and the ISOLDE Collaboration, Physica B 273-274, 144 (1999).

[13] D.W. Jenkins and J.D. Dow, Phys. Rev. B 39, 3317 (1989).

[14] T.L. Tansley and R. J. Egan, Phys. Rev. B 45, 10942 (1992).

[15] P. Boguslawski, E.L. Briggs, and J. Bernholc, Phys. Rev. B 51, 17255 (1995).

[16] J. Neugebauer and C.G. Van der Walle, Phys. Rev. B 50, 8067 (1994).

[17] R. Magerle, in: Defects in Electronic Materials II, ed. J. Michel, T. Kennedy, K. Wada, and K. Thonke, Met. Res. Soc. Sympos. Proc. Vol. 442, (Mater. Res. Soc., Pittsburgh, 1997), p.3.

[18] A.K. Viswanath, J.I. Lee, S. Yu, D. Kim, Y. Choi, and C. Hong, J. Appl. Phys. 84, 3848 (1998). 